\title{
Possibility of Cluster Strategy Use by Readers in Response to Filling the Blank Test
}

Parisa Daftarifard

Asst. Prof., Faculty of Persian Literature and Foreign Languages, South Tehran Branch, Islamic Azad University, Tehran, Iran, pdaftaryfard@azad.ac.ir

Tania Samadian

$\mathrm{PhD}$, Faculty of Persian Literature and Foreign Languages, South Tehran Branch, Islamic Azad University, Tehran, Iran, tnsamadian@yahoo.com

The present article reported an exploratory investigation into the nature of the reading comprehension strategies of English Foreign Language learners across proficiency levels. The aim of this study was to probe possible differences between elementary, intermediate, and expert readers' use of cluster strategy in the First Certificate English test. The collected data from 19 participants' Thinking Aloud on the gapped section of the test were analyzed both through content analysis and tabulation. The results indicated that readers at different language proficiency levels did not use a single profile strategy to answer a reading comprehension test item or to process a text, but rather used different combination or cluster strategies. The participants located in the intermediate language proficiency levels used more various strategy types to answer a single reading question than those who were located at the two extreme ends of the spectrum. Hence, focusing on teaching efficient cluster strategies rather than teaching single isolated strategies would be more productive in today's reading classrooms.

Keywords: first certificate of English (FCE), language proficiency levels, reading comprehension, strategy cluster, think-aloud protocols

\section{INTRODUCTION}

By far, one of the persistent problems in the actual practice of reading comprehension activity has been that of understanding the underlying processes that more or less proficient readers undergo to answer reading questions. Although it has been assumed that competent readers deploy efficient strategy to answer the reading questions (Cohen \& Upton, 2007), more recently scholars have doubted the idea of a single profile strategy in favor of cluster and combination strategies (Macaro, 2001). In other words, the choice of cluster strategy is claimed to be an indicator of readers' failure or success in a reading comprehension test (Graham, et al., 2010). Macaro (2001) believes that one single strategy might not determine the good or poor performance of respondents on a test; instead, poor performance might be the result of not being able to use "a full range of strategies in combination" (p. 264).

Literature is replete with research and studies conducted on the nature of Reading Comprehension (RC) processes to elucidate strategies efficient readers utilize to answer items in an RC test (Abbott, 2006; Alderson, 2000; Grabe, 2000). A clear picture of the nature of RC processes has implications for the way we prepare materials to teach RC, devise RC tests, and interpret the test results. Strategic use of language has frequently been referred to as one of the components of language competence, named strategic competence a proficient language user is believed to have (Bachman, 1990). Therefore, clarifying what the RC processes surely affects the way test scores are interpreted. As were 
expounded by Bachman and Palmer (1996), "[Unless] we can demonstrate that the inferences we make on the basis of language tests are valid, we have no justification for using test scores for making decisions about individuals" (p. 95).

The argument as to whether language users in general and readers in particular use single profile strategy or cluster of strategies has recently gained a growing popularity among scholars (Cohen, 2007; Graham \& Macaro, 2007; Graham, Santos, \& Vanderplank, 2010; Grenfell \& Macaro, 2007). For instance, Grenfell and Macaro (2007) believe that intermediate language users possibly deploy more efficient cluster strategies to answer reading items (Grenfell \& Macaro, 2007). To Cohen (2007), the use of cluster strategies indicates expert language users' consciousness, attentiveness, and goal orientation to learning. Accordingly, in his study, experts have concurred "strategic behavior could fall along a continuum from a single action to a sequence of actions" (p. 35). Also, they assert that there might be the possibility that more complex tasks require the use of cluster strategies which may vary at different proficiency levels. Considering the paucity of research on the new concept of strategy chains, it seems to be indispensable to investigate the patterns of possible cluster or chain strategies that both less and more proficient readers may orchestrate to answer the reading test items (Graham \& Macaro, 2007). As was mentioned earlier, research on reading and test-taking strategies is not new; however, the new trend is whether single strategies are responsible for learning to occur or strategies in combination (Macaro, 2007; Goh, 2019).

To bridge this gap, this study strives to investigate the possible reading test-taking cluster strategies, if existent, which might be responsible for elementary and intermediate readers' failure and achievement. Therefore, the present study seeks to determine if there is any trace of respondents' use of "cluster strategy" when choosing both the correct and wrong options in the second reading task of the new FCE test (from hereon, FCER). More specifically, the paper aimed to answer the following questions:

- Do expert readers use cluster strategies when answering the FCER gap-filling task?

- What are the cluster strategies that elementary and intermediate readers use to answer FCER gapfilling task correctly?

\section{REVIEW OF THE LITERATURE}

RC can be explained from three possible perspectives (Khalifa \& Weir, 2009): (a) factorial approach, (b) reading sub-skill approach, and (c) cognitive processing approach. In factorial approach, reading comprehension is divisible in nature and can be broken down into two or more components (Grabe, 2000). This interrelationship of the components, however, are explained either hierarchically (Hughes, 1990; Daftarifard \& Tristan, 2016; Weir, 1993), or in a parallel way (Rumelhart \& Ortony, 1977 as cited in Grabe, 1991).

According to the sub-skill approach, the skill and strategy approach to reading comprehension focuses on "behavioral aspect" of reading comprehension based on which good performance on reading is attributed to being competent on reading sub-skills. Theoretically speaking, strategies are defined as actions that are deliberately taken by learners to "make learning easier, faster, more enjoyable, more self-directed, more effective, and more transferable to new situations" (Oxford, p. 8). Anderson, Bachman, Perkins, Cohen, (1991) found that the use of strategies was task dependent. They found that there was a significant association between reading test taking, reading strategies and question types, between the frequency of strategy use and item discrimination and difficulty. They found that item difficulty is associated with skimming, paraphrasing, guessing, selecting through elimination, comprehending text, and connecting references to time, whereas item discrimination is associated with failure in understanding parts of the text. In contrast, inference type questions are associated with 
guessing, matching items with text, and interpreting whereas factual questions require understanding direct statements.

The cognitive processing model of reading comprehension explains reading in terms of three levels of representation. They are constructed as a result of the coordinated application of processes to reading types in order to attain two possible goals (expeditious reading versus careful reading).

FCE is an upper intermediate (B2) language exam (Weir \& Khalifa, 2008a, 2008b). At this level, learners are able to distinguish between main and supporting ideas. According to Weir and Khalifa (2008a), FCE measures the following types of comprehension:

- comprehension across sentences

- comprehension of the overall text

- scanning and searching the reading

- $\quad$ searching the reading

The FCER (2000) has taken both cognitive and skill approach to reading comprehension (Khalifa \& Weir, 2009). The test is designed to measure examinees' competency at the intermediate level (Hawkey, 2009). The FCER (2002) aims at measuring examinee's ability to scan the text for relevant information, understand the general meaning of content, and capture the most important ideas. The test has three parts with the total length of 2000 words. It draws on thirty questions representing several local and global reading types such as comprehending across sentences, scanning overall text, and searching reading. The FCER incorporates approximately 550-700 words per text. Furthermore, the texts include the selection of three possible text types, classified by text function: (1) referential, (2) emotive, and (3) poetic (Khalifa \& Weir, 2009). Most importantly, different parts of the reading section are expected to elicit different cognitive processing to be comprehended. The first part, representing multiple choice formats, calls for strategies to find main ideas, writers' attitude, tone, purpose, and opinion, to understand the propositional meaning, and text organization features. The second part, gapped text format, requires that readers understand the text structures at the global level. The third part, representing multiple matching formats, is expected to call for readers' ability to search for specific information within multiple texts. This article aims at exploring possible cluster strategies that both elementary and intermediate learners might deploy when answering the second part of FCER test, that is, gapped text format.

\section{METHOD}

Due to the research purpose a descriptive methodology was chosen as the research paradigm. The think-aloud analysis was used to discern hidden strategies the test takers chose to answer reading items. The concurrent think-aloud reports method (Caldwell \& Leslie, 2010) was chosen because the respondents generated their thoughts while answering the questions.

\section{Participants}

Nineteen participants, forming three groups across language proficiency, took part in this study. The first two groups included 16 (12 female and 4 male) Iranian university students majoring in English and the last group included 3 expert ( 2 female and 1 male) Ph.D. holder readers in TEFL who had been teaching English at university for more than a decade. All volunteers were aged between 20 and 38 years old.

To differentiate between elementary, intermediate, and advance readers; the participants were given the Oxford Placement Test and the FCER. The results revealed three groups across proficiency: 
Elementary readers (poor readers, $n=8)$, Intermediate readers $(n=8)$, and the expert group $(n=3)$. The expert group were equivalent to $\mathrm{C} 1$ language proficiency level based on Common European Framework of Reference (CEFR) criteria.

Table 1 shows the participants' performance on three reading tasks of FCER. The result of the second task will be specifically reported in this paper.

Table 1

Descriptive Statistics of Elementary and -Intermediate Performance

\begin{tabular}{lll}
\hline Features & Elementary & Intermediate \\
Number of Students & $8^{*}$ & 8 \\
Task 1 (Mean score**) & 1.6 & 4.3 \\
SD & 0.9 & 1.8 \\
SEM & 0.3 & 0.6 \\
\hline Task 2 (Mean score) & 2 & 5.6 \\
SD & 1 & 1 \\
SEM & 0.3 & 0.4 \\
\hline Task 3 (Mean score) & 6.7 & 11 \\
SD & 1.8 & 2.2 \\
SEM & 0.6 & 0.8 \\
FCER Mean score & 10.5 & 20.5 \\
SD & 2.3 & 2.9 \\
SEM & 0.8 & 1.05 \\
\hline Note: $*$ see appendix for CEFR Specification ** Task 1 total possible score was 8; Task 2 total
\end{tabular}
possible score was 7; Task 3 total possible score was 15

\section{Instruments}

Three instruments were used in this study: (a) Oxford Placement Test (OPT), (b) the FCER (Cambridge University Press 2010), and (c) coding system. Oxford Quick Placement new version test (2001, the version I) was used to determine the participants' English language proficiency level in terms of The Common European Framework of Reference (CEFR). The CEFR has been developed through a progressive process of scientific research and is the benchmark against which the results of all high-stake standard tests like TOEFL, IELTS, and the like are interpreted.

The FCER was used to elicit think aloud analysis for the purpose of this study. The FCER draws on thirty items representing three comprehension tasks (Multiple choices, fill in the blank, and multiple matching tests) each of which is claimed to measure different reading abilities at the intermediate level specified by CEFR. Multiple choice items aim to measure global careful reading whereas the fill in the blank section is assumed to measure textual organization at the level of global reading and part three aims at measuring learners' ability to search at both local and "occasionally" global level (Khalifa \& Weir, 2009, p. 65). For the sake of space, however, in this article, only the participants' performance on the second reading task of FCE is reported.

The third instrument that was used in the present research was the coding system; nine strategies were agreed upon in the present research. The first strategy derived was Option Based Reading Selection or OPS (Cohen, 1998). An OPS occurs when the test taker selects the answer initially based on the options the item suggests. i.e. distracters and correct response. The next strategy was External Consistency or EXC (Baker, 1989) which occurs when the readers check the idea with their own experiences. Paraphrasing or PARA, was the third extracted strategy, which refers to a propositional understanding of the sentence (Nuttall, 1996). Internal Consistency or Text Based Model (TBM) was the next extracted strategy which refers to readers' ability to gradually construct a semantic network through connecting sentences together (Baker, 1989). 
The fifth elicited strategy was Understanding Text based on Text Structure (TBS). This strategy refers to understanding meaning through determining the relationship between each text section and heading, understanding text organization such as exemplification, cause and effect, etc., recognizing word relationship (cohesive ties), and recognizing the function of punctuation like appositive (Grabe 2009; Carrell, 1990; Trimble, 1985). Still another strategy was extracted named as Recalling or REC in which memory is used to answer the questions (Alderson, 2000, Grabe, 2009). The seventh extracted strategy was Word-based reading or WBT (Cohen, 1998). It is a kind of strategy through which the readers are highly dependent on the words used in either the stem or the text as the sole source of information to answer the question based on (Nakatani, 2006). Inferencing or INF was another elicited strategy or reading beyond the line (Alderson, 2000). And finally, some readers might plan for their reading activity or evaluate their progress; this strategy is categorized as metacognitive strategy or Evaluation or EV (Carrell, 1989; Imtiaz, 2004; Schraw \& Dennison,1994).

\section{Procedure}

The data were collected by the author who met the participants individually. At the beginning of the study, participants were given the Oxford Placement Test to be grouped into elementary, intermediate, and expert readers. To familiarize the students with the "think-aloud" protocol, the first session began with a ten-minute training think-aloud procedure while the examiner answered the reading questions similar to that of FCE. The following instruction was written for the participants and explained further when required; "While you are answering each question, talk aloud your thoughts and explain the reason for choosing the correct answer for each question."

Then the participants practiced think aloud while answering the same reading questions and expressed the reason and described how they came up with the answers they chose. The researcher indicated that the procedure of thinking aloud might seem impractical at first, but eventually, with practice, the participants enjoyed doing it. After the training session, the participants were told to apply the same procedure to three reading tasks of the FCER from which only the second reading task is reported in this paper. Depending on their reading proficiency, readers took 1 to 2 hours to answer the questions, along with thinking aloud about how they reached the answer at IAU, South Tehran Branch. They recorded their thoughts while trying to match the appropriate sentence to the gapped text.

The participants' voices were recorded for further analysis. The participants were asked to express themselves through their mother tongue to ensure that all the students were capable of performing the talk-aloud task. In the next step, the participants' taped think-aloud protocols were transcribed into 9532-word reports. The elicited reports on the FCER was coded using the checklist prepared on reading test taking strategies based on an in-depth review of the literature and data (Janssen, Braaksma, \& Rijlaarsdam, 2006; Macaro \& Mutton, 2009; Macaro, 2007; Phakiti, 2003).

Two raters who were familiar with both think-aloud and coding system coded the data. The second rater who did not participate in this study coded $50 \%$ of the protocols independently to ensure reliability and consistency of the coding system. While coding the data, two raters modified the list to meet the features of the data. The sources of inconsistency were four reading strategies as (a) constructing mental model; (b) summarizing a text; (c) inferring the meaning, and (d) guessing the meaning of unknown words. To increase consistency, we combined the mental model and summarized the text under the title of internal consistency. Inference and guessing the meaning of the unknown words were grouped under the title of inferencing. Furthermore, Speed Reading as a possible demonstrated strategy was omitted for both competent and elementary readers. The strategies were modified to meet the data features of the study. They were reduced to nine strategies each of which was explained briefly in section 2.2. After achieving 90\% agreement on the coding system between the rater and the researcher, the researcher coded the remainder of the think-aloud protocol data individually. 


\section{FINDINGS}

The frequency of the coded data was tabulated to check whether there was any cluster strategies in the elementary and intermediate participants' responses to the second task of the FCER and how they were different from the performance of the expert readers on the test. First the data, in general, will be shown and discussed, and then the single possible responses for each of elementary, intermediate, and expert readers will be compared.

\section{Expert Readers: Single or cluster Strategy}

To answer the first question of the present study, "Do expert readers use cluster strategies when answering the FCER gap-filling task?", the gapped text of FCER was given to three experienced Ph.D. participants majoring in TEFL to elicit their think-aloud episodes. Furthermore, this stage was necessary for two purposes: one was to determine the function of each item or gap in this part of FCER, and the other was to compare experts' underlying processes with those of elementary and intermediate readers. Expert readers were used as a benchmark to compare other readers at different language proficiency levels.

In the present research, experts' think aloud was used instead of experts' judgment due to potential problems that the nature of judgment might cause (Alderson, 1990). Research has shown that judging and naming items might not lead experts to an agreement. In this connection, Tavakoli and Barati (2011) found no significant agreement among Iranian expert raters on what FCER items might measure. This is in line with other scholars' (Alderson, 1990; Lumley, 1993; Weir, 1993) finding that there is less consistency among experts' judgment that what the item is measuring. A possible explanation could be that no single strategy would lead them to answer the reading item.

The coded data showed that from 21 possible responses for using strategy ( 7 items multiplied by 3 experts), 9 answers (40\%) were correct based on using cluster strategy Inter- rater's agreement on 7 items was considerably high (Alpha $=0.9$ ). The total strategies experts agreed on is summarized in Table 2. However, as is shown in this table, Experts mostly went through single strategy to answer the item correctly. Note that only the used strategies have appeared in this table.

Table 2

\begin{tabular}{|c|c|c|c|c|c|c|c|c|c|}
\hline $\mathrm{Q}$ & Expert Name & OPS & EXC & PARA & TBM & INF & TBS & Single Strategy & Strategy cluster \\
\hline 1 & 1 & 1 & 0 & 0 & 0 & 0 & 1 & & $*$ \\
\hline 1 & 18 & 1 & 0 & 0 & 1 & 0 & 1 & & $*$ \\
\hline 1 & 33 & 0 & 0 & 1 & 0 & 0 & 1 & & * \\
\hline 2 & 1 & 0 & 0 & 0 & 1 & 0 & 1 & & * \\
\hline 2 & 18 & 0 & 0 & 1 & 0 & 0 & 0 & * & \\
\hline 2 & 33 & 0 & 0 & 1 & 1 & 0 & 0 & & * \\
\hline 3 & 1 & 0 & 0 & 0 & 0 & 1 & 0 & * & \\
\hline 3 & 18 & 0 & 0 & 0 & 1 & 0 & 0 & * & \\
\hline 3 & 33 & 0 & 0 & 0 & 1 & 1 & 0 & & $*$ \\
\hline 4 & 1 & 0 & 0 & 0 & 1 & 0 & 1 & & * \\
\hline 4 & 18 & 0 & 0 & 0 & 1 & 0 & 0 & * & \\
\hline 4 & 33 & 0 & 0 & 0 & 1 & 0 & 0 & * & \\
\hline 5 & 1 & 0 & 0 & 0 & 0 & 0 & 1 & * & \\
\hline 5 & 18 & 0 & 0 & 0 & 1 & 0 & 0 & * & \\
\hline 5 & 33 & 0 & 0 & 0 & 0 & 0 & 1 & $*$ & \\
\hline 6 & 1 & 0 & 0 & 0 & 0 & 0 & 1 & * & \\
\hline 6 & 18 & 0 & 0 & 0 & 0 & 0 & 1 & * & \\
\hline 6 & 33 & 0 & 0 & 0 & 1 & 0 & 0 & $*$ & \\
\hline 7 & 1 & 0 & 0 & 0 & 1 & 0 & 1 & & $*$ \\
\hline 7 & 18 & 0 & 0 & 0 & 1 & 0 & 1 & & $*$ \\
\hline 7 & 33 & 0 & 0 & 0 & 1 & 0 & 0 & $*$ & \\
\hline
\end{tabular}


Note: OPS: Option Based Selection, EXT (External Consistency), PARA (Paraphrasing), TBM (Text based Mental model or internal consistency), INF (Inference), And TBS (TEXT Structure-based understanding)

The result indicated that the expert readers' frequently used strategies were TBM and TBS. The least frequent strategies were Paraphrasing and INF. Furthermore, EXT and OPT were not used at all.

\section{Elementary and Intermediate Readers' use of cluster Strategy in correct answer choice}

To answer the second question of this study, "what cluster strategies do elementary and intermediate readers use to answer FCER gap-filling task correctly?" the data were summarized using Custom Table method in SPSS. Custom Tables enable researchers to categorize data into different vertical and horizontal layers. This feature enables users to define layers within two dimensions. The result is summarized in the Table 3 which shows the cross frequencies of defined variables. The frequencies of extracted strategies were compared across language proficiency and items. Table 3 shows the strategy clusters for each item in both groups (elementary as well as intermediate readers). Each group consists of 8 participants.

Table 3

Elementary and Intermediate Readers Strategy cluster when the Answer is Correct

\begin{tabular}{|c|c|c|c|c|c|c|c|c|c|c|}
\hline \multicolumn{2}{|c|}{ LEVEL } & \multicolumn{9}{|c|}{ Strategy Type } \\
\hline \multirow{2}{*}{\multicolumn{2}{|c|}{ ITEMS }} & OPS & EXT & PARA & TBM & INF & TBS & REC & WBT & EV \\
\hline & & Count & Count & Count & Count & Count & Count & Count & Count & Count \\
\hline \multirow{7}{*}{ 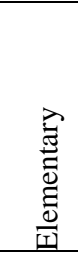 } & 1 & 0 & 0 & 2 & 2 & 1 & 0 & 0 & 0 & 0 \\
\hline & 2 & 0 & 0 & 1 & 1 & 0 & 0 & 0 & 1 & 0 \\
\hline & 3 & 1 & 0 & 1 & 1 & 0 & 0 & 0 & 1 & 0 \\
\hline & 4 & 0 & 0 & 1 & 1 & 0 & 0 & 0 & 1 & 0 \\
\hline & 5 & 0 & 0 & 0 & 0 & 0 & 1 & 0 & 0 & 0 \\
\hline & 6 & 0 & 0 & 0 & 2 & 1 & 0 & 0 & 1 & 0 \\
\hline & 7 & 1 & 0 & 0 & 0 & 0 & 0 & 0 & 1 & 0 \\
\hline & & OPT & EXT & PARA & TBM & INF & TSB & REC & WB & EV \\
\hline \multirow{7}{*}{ 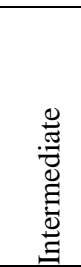 } & 1 & 5 & 0 & 2 & 5 & 0 & 2 & 1 & 2 & 2 \\
\hline & 2 & 4 & 1 & 2 & 5 & 0 & 1 & 0 & 3 & 0 \\
\hline & 3 & 4 & 1 & 1 & 6 & 0 & 4 & 0 & 1 & 0 \\
\hline & 4 & 2 & 0 & 2 & 3 & 0 & 1 & 0 & 1 & 0 \\
\hline & 5 & 2 & 0 & 2 & 2 & 0 & 4 & 0 & 0 & 2 \\
\hline & 6 & 1 & 0 & 0 & 3 & 3 & 0 & 0 & 2 & 0 \\
\hline & 7 & 2 & 0 & 0 & 2 & 1 & 1 & 0 & 3 & 1 \\
\hline
\end{tabular}

Note: OPS: Option Based Selection, EXT (External Consistency), PARA (Paraphrasing), TBM (Text based Mental model or internal consistency), INF (Inference), TSB (TEXT Structure-based understanding), REC (Recalling information), WB (Word-based reading), EV (evaluation as metacognitive strategy)

As is shown in this table, elementary readers used strategies considerably less than intermediate readers. There are 56 possible responses (number of items multiplied by the number of participants). As is shown here, about $59 \%$ of answers to items were done through single profile strategy. Therefore, only $41 \%$ of responses were answered using cluster strategies.

Moreover, to demonstrate who used cluster strategies in answering these 7 items of the gapped text, the data were tabulated into Table 4, and the performance of those with the scores more than 4 were compared. Table 4 demonstrates the profile of those test takers at two language proficiency levels of elementary and intermediate with the total score more than or equal to 4 on the 7 item gapped text task. As is shown in this table, "X" shows that the test takers used single profile strategy to reach the correct answer, whereas " $\checkmark$ " shows that the test takers used cluster strategies to reach the correct 
answer. To understand the difference, chi-square was run to examine if there is any quantitative significant difference among the patterns of responses. As can be seen in Table 4, there are 63 observations (number of items multiplied by the number of participants. From among this number of 21 correct responses were based on the use of strategy cluster and 23 responses were based on single profile strategy (Chi-Square $(3)=10.33$, p < 0.05). This shows that language users were dynamic in choosing a single profile or a cluster of strategies in responding the items correctly.

Table 4

Test Takers' Profile who answered the Items with Strategy Cluster at Elementary and Intermediate Language Proficiency Levels

\begin{tabular}{|c|c|c|c|c|c|c|c|c|c|}
\hline \multirow[t]{2}{*}{ LEVEL } & \multirow{2}{*}{$\begin{array}{l}\text { Test } \\
\text { Takers' } \\
\text { Name }\end{array}$} & \multicolumn{7}{|c|}{ Items } & \multirow{2}{*}{$\begin{array}{l}\text { Total } \\
\text { score }\end{array}$} \\
\hline & & 1 & 2 & 3 & 4 & 5 & 6 & 7 & \\
\hline \multirow[t]{6}{*}{ Elementary } & 13 & + & + & $\checkmark$ & $\mathrm{X}$ & $X$ & $\mathrm{O}$ & $\checkmark$ & 4 \\
\hline & 15 & $X$ & + & $\checkmark$ & $\mathrm{X}$ & $X$ & + & + & 4 \\
\hline & 25 & $\checkmark$ & $X$ & $\checkmark$ & $\checkmark$ & $\mathrm{O}$ & $X$ & $X$ & 6 \\
\hline & 29 & + & $\checkmark$ & $\checkmark$ & $\checkmark$ & $\checkmark$ & + & $\checkmark$ & 5 \\
\hline & 2 & $\mathrm{X}$ & $\mathrm{O}$ & $\checkmark$ & + & $\mathrm{X}$ & $X$ & $\mathrm{X}$ & 5 \\
\hline & 3 & $\mathrm{O}$ & $X$ & $\mathrm{O}$ & $\mathrm{X}$ & $\mathrm{X}$ & $\checkmark$ & $\mathrm{O}$ & 4 \\
\hline \multirow[t]{4}{*}{ Intermediate } & 5 & $X$ & $\mathrm{O}$ & $\checkmark$ & $\mathrm{X}$ & $\mathrm{O}$ & $\mathrm{X}$ & $\checkmark$ & 5 \\
\hline & 14 & $\checkmark$ & $X$ & $\checkmark$ & $\checkmark$ & $X$ & $X$ & $\checkmark$ & 7 \\
\hline & 23 & $\checkmark$ & $\checkmark$ & $\checkmark$ & $\checkmark$ & $X$ & $\checkmark$ & $\checkmark$ & 7 \\
\hline & 31 & + & + & X & + & X & $\checkmark$ & $\checkmark$ & 4 \\
\hline
\end{tabular}

Note: * "X" shows that the test takers used single profile strategy to reach the correct answer ; " $\checkmark$ " shows that the test takers used strategy cluster to reach the correct answer; " 0 " shows that the test takers used single profile strategy to reach the wrong answer; "+" shows that the test takers used strategy cluster to reach the wrong answer

In order to investigate the possibility of cluster strategies use instead of single strategy, the data frequency was checked (See Table 5). As is shown in Table 5, most of the language users used cluster strategies when answering the items correctly except for lower intermediate group (B1). Expert participants' only four response were incorrect, and it happened when the single profile strategy was used.

Table 5

Percentage and Frequency of Strategy Type across Language Proficiency Levels

\begin{tabular}{llll}
\hline Level & Answer & Strategy Type & Frequency (Percentage) \\
\hline A2 & Correct & Single Profile & $15(46.9 \%)$ \\
& & Strategy Cluster & $17(53.1 \%)$ \\
& Incorrect & Single Profile & $25(55.6 \%)$ \\
& & Strategy Cluster & $20(44.4 \%)$ \\
\hline B1 & Correct & Single Profile & $17(65.4 \%)$ \\
& & Strategy Cluster & $9(34.6 \%)$ \\
& Incorrect & Single Profile & $40(69 \%)$ \\
& Correct & Single Profile & $18(31 \%)$ \\
\hline B2 & Incorrect & Strategy Cluster & $29(65.4 \%)$ \\
& & Single Profile & $4(36.4 \%)$ \\
& Correct & Strategy Cluster & $7(63.6 \%)$ \\
\hline C & Single Profile & $14(58.3 \%)$ \\
& & Strategy Cluster & $10(41.7 \%)$ \\
& & Single Profile & $4(100 \%)$ \\
\hline
\end{tabular}


In the Elementary group, from 56 possible responses (number of items multiplied by the number of participants) for using reading strategy, 33 responses (59\%) were answered by a single strategy which led to an incorrect response. Among these participants, only one test taker answered all questions through using single profile strategy. This participant gained 2 out of 7 in this task. On the other hand, from 56 possible responses, intermediate readers used cluster of strategies by $78.5 \%$. In case they used single strategy (12 responses), only two responses were wrong. This shows that intermediate readers employed more strategies to solve a reading problem. This finding delineates that if elementary readers deploy only a single strategy, they have the least chance to answer the reading item of this nature correctly.

\section{CONCLUSION, DISCUSSION AND SUGGESTIONS}

This study aimed to explore the preference of cluster strategies use in lieu of a single profile strategy use of Iranian EFL readers. Specifically, it attempted to investigate the possible different cluster strategies pattern of elementary and intermediate readers when answering the second reading task of FCE test. To make an in-depth investigation of readers' actual and possible strategy use during reading processes (Green, 1998) the think-aloud method was used.

In sum, consistent with some scholars' claim that a full range of strategies in combination, might be responsible for language users' success or failure in comprehending language (Cohen, 2007; Macaro, 2001), findings of this study also indicated that both elementary and intermediate readers mostly resorted to cluster strategy use when they answered the gapped reading task of the FCE. It was observed that intermediate readers most frequently used a combination or cluster strategies. Li (1992) working on reading items found that test takers would not use a single strategy to answer test items; nor would they follow the strategy the test item intended to measure. Huang's (2018) research revealed that L2 Chinese readers grouped multiple strategies to understand a Chinese text.

The results of this study indicated that elementary readers mostly failed to succeed in answering a question correctly when they used a single strategy to respond to a question. In contrast, intermediate readers used a variation in the number and nature of strategy and tended to use strategy in combination. Readers in this group actively searched the overall meaning of the text, along with text structure. In addition, they paid attention to other alternatives to answer the item when facing difficulty. This variation in the number and nature of strategy is well supported by Wade (1990)

Another finding of this study was that not only readers' choice of strategy affected reading comprehension of this type, but also the way readers deciphered the propositional meaning of sentences illustrated their cluster strategy pattern use. For example, Case 30's used of strategy combination sounded logical, but his answer was incorrect; he incorrectly paraphrased and, accordingly, constructed the mental model of the text. This is in line with other studies Meyers, Lytle, Palladino, Devenpeck, \& Green, 1990; Caldwell \& Leslie, 2010) that revealed correct deciphering propositional and hidden meaning of the text is indicative of correct comprehension.

The outcome of the present research indicated that the development in reading processes along with language proficiency is not steady and straightforward but rather curved. This is similar to what McLaughlin (1987) theorized under the concept of restructuring. Restructuring implies that interlanguage development is not linear but in the form of a $U$ shape. Elementary readers use strategies which tend to be simple, single, and less correlative with the purpose of the test. They use more textbased than situation-based strategies. In contrast, with the increase in language proficiency levels, reading processes are more complicated in nature and number, and intermediate users tend to use strategy in combination or a cluster of strategies. However, proficient language users and experts read the information with ease and few numbers of strategies. In other words, strategy use development can 
be portrayed as a bell-shaped format with elementary and experts placing on the two extreme sides and intermediate students forming the central parts of it with the highest strategy clusters.

To Cohen (1998), the limited number of strategies in response to an item might indicate respondents' control over the item, assuming that the selected strategies are efficient and well-chosen. Elsewhere, Barati and Kashkoul (2013) worked on 70 EFL undergraduate university students who were varying in their language proficiency. The variation in strategy use was significantly more in intermediate learners taking the reading section of FCE. In the same line, Huang (2018) found out that readers' strategy choice was affected by their language proficiencies. This means that the more proficient L2 Chinese readers grouped multiple strategies to understand a Chinese text.

Another finding of this study was that elementary, intermediate, and expert readers used different cluster strategies or strategy in combination. Elementary readers mostly relied on words than the whole sentence. This finding is in compliance with the research of McGuire and Yewchuk (1996) who found that word-based readers failed to paraphrase the sentences correctly. The findings of the present research showed the flexibility of less and more expert language readers in using single profile strategy or cluster of strategies to answer reading questions. It seems that readers at elementary and expert levels are less strategic than intermediate level readers. This only seems logical; as learners improve their language, they seem to have more self-regulation and self-talk. Hence the result of this study emphasizes the importance of inclusion of "self-explanation" techniques in language teaching especially when the teachers' focus is to ameliorate the language processing (McNamara \& Magliano, 2009).

The possibility of strategy combination or cluster use is one of the most recent debates in second language acquisition. Much research studies using think-aloud analysis indicate that the use of a single strategy is not responsible for neither failure nor success of language readers. The results of this study extend previous literature (e.g. Wade, 1990) in which it was shown that one strategy type might be effective for one reader but not necessarily for another. More researches about the possibility of using cluster strategies instead of a single strategy in solving a reading task are needed to provide a better understanding of EFL perception, and to yield useful pedagogical implications to improve the EFL reading curriculum, as well as teacher training courses.

\section{REFERENCES}

Abbott, M. (2006). ESL reading strategies: Differences in Arabic and Mandarin speaker test performance. Language Learning, 56(4), 633-670. https://doi.org/10.1111/j.1467-9922.2006. 00391.x

Alderson, J. C. (1990). Testing reading comprehension skills (part one). Reading in a Foreign Language, 6(2), 425-438.

Alderson, J. C. (2000). Assessing reading. Cambridge: CUP.

Anderson, N. J., Bachman, L., Perkins, K., Cohen, A. (1991). An exploratory study into the construct validity of a reading comprehension test: triangulation of data sources. Language Testing, 8(1), 41-66.

Bachman, L. F. (1990). Fundamental considerations in language testing. Oxford: OPT

Bachman, L. F., \& Palmer, A. (1996). Language testing in practice. Oxford: Oxford University Press.

Baker, L. (1989). Metacognition, comprehension monitoring and the adult reader. Educational Psychology Review, 1(1), 3-38.

Barati, H., \& Kashkoul, Z. (2013). Test-taking strategies and task-based assessment: The case of Iranian EFL learners. IJRELT, 1(2), 111-129. doi: 10.5861/ ijrsll.2014.852. 
Caldwell, J., \& Leslie, L. (2010). Thinking aloud in expository text: Processes and outcomes. Journal of Literacy Research, 42, 308-410. doi: 10.1080/1086296X.

Carrell, P. L. (1989). Metacognitive awareness and second language reading. The Modern Language Journal , 73(ii), 121-134.

Carrell, P. L. (1990). Awareness of text structure: Effects on recall. Language Learning, 42(1), 1-20.

Cohen, A.D. (1998). Strategies and processes in test taking and SLA. In L. F. Bachman, \& D. Cohen (Eds.), Interface between second language acquisition and language testing research (pp. 90-111). Cambridge: Cambridge University Press.

Cohen, A. D. (2007). Coming to terms with language learner strategies. In A. D. Cohen, \& E. Macaro (Eds.), Language learner strategies: Thirty years of research and practice (pp. 29-46). Oxford: OUP.

Cohen, A. D., \& Upton, Th. A. (2007). I want to go back to the text: Response strategies on the reading subtest of the new TOEFL. Language Testing, 24(2), 209-250. doi:10.1177/0265532207076364.

Daftarifard, P., \& Tristan, A. (2016). Implicational scaling of reading comprehension construct: Is it deterministic or probabilistic? Journal of English Language Pedagogy and Practice, 9(18), 118-142.

Goh, J. X. (2019) A study on the use of different reading strategies to read English text in Malaysian ESL classroom. Final Year Project, UTAR. Retrieved from http://eprints.utar.edu.my/3500/.

Grabe, W. (1991). Current developments in second language reading research. TESOL Quarterly, 25(3), 375-406.

Grabe, W. (2000). Reading research and its implications for reading assessment. In A. Kunnan (Ed.), Fairness and validation in language assessment (pp. 226-260). Cambridge: CUP.

Graham, S., \& Macaro, E. (2007). Designing Year 12 strategy training in listening and writing: From theory to practice. Language Learning Journal, 35, 153-173.

Graham, S., Santos, D., \& Vanderplank, R. (2010). Strategy clusters and sources of knowledge in French L2 listening comprehension. Innovation in Language Learning and Teaching, 4(1), 1-20. doi: https://doi.org/10.1080/09571730701599203.

Green, A. (1998). Verbal protocol analysis in language testing research. Cambridge: CUP.

Grenfell, M., \& Macaro, E. (2007). Claims and critiques. In A. D. Cohe,n \& E. Macaro (Eds.), Language learner strategies: Thirty years of research and practice (pp. 9-28). Oxford: OUP.

Hawkey, R. (2009). Examining FCE and CAE: Key issues and recurring themes in developing the first certificate in English and certificate in expert English exams. Cambridge: Cambridge University.

Huang, S. (2018). Effective strategy groups used by readers of Chinese as a foreign language. Reading in a Foreign Language, 30(1), 1-28.

Imtiaz, Sh. (2004). Metacognitive strategies of reading among ESL Learners. South Asian Language Review, XIV (1 \& 2), 34-43. doi: 10.5539/elt.v9n3p117.

Janssen, T., Braaksma, M., \& Rijlaarsdam, G. (2006). Literary reading activities of good and weak students: A think aloud study. European Journal of Psychology of Education, XXI(1), 35-52. doi: 10.1007/BF03173568.

Khalifa, H., \& Weir, C.J. (2009). Examining reading: Research and practice in assessing second language reading. Studies in Language Testing 29, Cambridge: UCLES/CUP 
Li, J. (2019). Development and validation of second language online reading strategies inventory. Computers and Education, 1-46. https://doi.org/10.1016/j.compedu.2019.103733.

Lumley, T. (1993). The notion of sub-skills in reading comprehension tests: an EAP example. Language Testing, 10(3), 211-234.

Macaro, E. (2001). Analyzing student teachers' code-switching in foreign language classroom: theories and decision making. The Modern Language Journal, 85, 531-548.

McNamara, D. S., \& Magliano, J. P. (2009). Self explanation and Metacognition: The dynamics of reading. In D. J. Hacker, J. Dunlosky, \& A. C. Graesser (Eds.), Handbook of Metacognition in education (pp.60-82). New York: Routledge.

McGuire, K.L., \& Yewchuk, C. (1996). Use of metacognitive reading strategies by gifted learningdisabled students: An exploratory study. Journal for the Education of the Gifted, 19, 293-314. doi: $10.1177 / 016235329601900304$.

McLaughlin, B. (1987). Theories of second-language learning. London: Edward Arnold.

Meyers, J., Lytle, S., Palladino, D., Devenpeck, G., \& Green, M. (1990). Think-aloud protocol analysis: An investigation of reading comprehension strategies in fourth-and fifth-grade students. Journal of Psychoeducational Assessment, 8(2), 112-127.

Nakatani, Y. (2006). Developing an oral communication strategy inventory. The Modern Language Journal 90(6), 151-168. doi: 10.1111/j.1540-4781.2006.00390.x.

Nuttall, C. (1996). Teaching reading skills in a foreign language. Hong Kong: Macmillan.

Oxford, R. L. (1990). Language learning strategies: What every teacher should know. MA: Heinle.

Oxford, R. L., \& Schramm, K. (2007). Bridging the gap between psychological and sociocultural perspectives on L2 learner strategies. In A. D. Cohen, \& E. Macaro (Eds.), Language learner strategies: Thirty years of research and practice (pp. 47-68). Oxford: OUP.

Schraw, G., \& Dennison, R. S. (1994). Assessing metacognition aswareness. Contemporary Educational Psychology, 19, 460-475. doi: 10.1007/BF02212307.

Tavakoli, E., \& Barati, H. (2011). Investigating the construct validity of the FCE reading paper in Iranian EFL context. Journal of Language Teaching and Research, 2(1), 239-247.

Trimble, L. (1985). English for science and technology: A discourse approach. Cambridge: Cambridge University.

Wade, S. (1990). Using think alouds to assess comprehension. The Reading Teacher, 43(7), 442-451.

Weir, C. J. (1993). Understanding and developing language tests. London: Prentice Hall.

Weir, C. J., \& Khalifa, H. (2008a). A cognitive processing approach towards defining reading comprehension. Studies in Language Testing, 29, 2-10. Cambridge: UCLES/CUP.

Weir, C. J., \& Khalifa, H. (2008b). Applying a cognitive processing model to main suite reading papers. Studies in Language Testing, 29, 11-16). Cambridge: UCLES/CUP.

Azhar M. (2007) Level of professionalisme among Islamic education trainees in practical teaching (Unpublished master thesis). Universiti Teknologi Malaysia. 